\title{
ABA Increases Susceptibility of Pepper Fruits to Infection of Anthracnose by Collectotrichum acutatum
}

\author{
Sookyeong Hwang ${ }^{1}$, Joohyung Kim ${ }^{1}$, Young Ho Kim ${ }^{2}$ and Heung Tae Kim ${ }^{1 *}$ \\ ${ }^{1}$ Department of Plant Medicine, Chungbuk National University, Cheongju, Chungbuk 361-763, Korea \\ ${ }^{2}$ Department of Agricultural Biotechnology, Seoul National University, Seoul 151-742, Korea \\ (Received on November 4, 2008; Accepted on November 20, 2008)
}

To examine the relationship between plant hormones and the development of pepper anthracnose, we investigated the effects of several plant hormones on the progression of disease symptoms. Of the five plant hormones examined, abscisic acid (ABA) increased the lesion length and disease incidence on detached fruits of Capsicum annuum cv. Nokkwang. The simultaneous application of ABA with inoculation of Colletotrichum acutatum JC24 resulted in increased lesion length, depending the concentration of $\mathrm{ABA}$ applied. Additionally, application of ABA caused the development of pepper anthracnose in fruits of Capsicum baccatum cvs. PBC80 and PBC81, which were previously resistant to the disease. Furthermore, ABA administration rendered increased pathogenicity of other isolates of $C$. acutatum BAC02063, PECH10, and TCBNU3 obtained from the Chinese matrimony vine, peach, and tea tree, respectively. Our data suggest that exogenous ABA may result in the suppression of defense mechanisms of pepper fruits against anthracnose, which leads to a change in the susceptibility of pepper fruits and the development of pepper anthracnose.

Keywords : abscisic acid, Colletotrichum acutatum, pepper anthracnose

Infection by fungal pathogens results in changes in host plant physiology as well as mechanical and biochemical disruptions. In the case of some plant pathogens such as Botrytis cinerea, the susceptibility of plant organs, particularly fruits and flowers, increases with ageing and ripening. Plant hormones that accelerate senescence, such as ethylene, tend to increase susceptibility, while those that delay senescence, such as cytokinin and gibberellin, tend to increase resistance (Elad, 1988, 1997).

Because plant development and responses to environmental cues are highly regulated by plant hormones, manipulation of hormone signals in host plants might create suitable environments for pathogen growth and reproduc-

\footnotetext{
*Corresponding author.

Phone) +82-43-261-2556, FAX) +82-43-271-4414

E-mail)htkim@cbnu.ac.kr
}

tion. Indeed, certain plant pathogens are capable of producing plant hormones (Lahey et al., 2004; Tudzynski, 2005). Biosynthesis of the plant hormone gibberellin was originally observed in Fusarium species to cause rice bakanae disease (Malonek et al., 2005). Overproduction of other plant hormones such as auxin and cytokinin by certain bacterial pathogens is the basis for tumor and gall formation in host plants (Escobar et al., 2001). Maor et al. (2004) recently reported in planta production of the auxin indole3-acetic acid (IAA) by Colletotrichum gloeosporioides; $C$. gloeosporioides produces most of its IAA by the indole-3aceteamide (IAM) pathway, which is absent in the host plant (Robinson et al., 1998). Interestingly, significantly higher amounts of IAA were produced by C. gloeosporioides during the biotrophic phase of the infection than during the necrotrophic phase (Maor et al., 2004). These results may indicate that IAA production plays an important role in fungi by jamming plant defense mechanisms, thereby causing hormone imbalances at the early infection stage.

Some plant pathogens successfully induce disease via their ability to suppress the inducible defense responses of their hosts. However, knowledge of the mechanisms of defense repression caused by fungal pathogens and the chemical nature of the responsible suppression is limited. The saponoin degradation product $\beta 2$-tomatine mediates the suppression of defenses in tomato plants by interfering with fundamental signal transduction processes (Bouarab et al., 2002; Martin-Hernandez et al., 2000). Supprescine A and $\mathrm{B}$, which are secreted by the pea pathogen Mycospherella pinoides, cause a delay in the elicitor response or $P A L$ transcription and a delay in the accumulation of pisatin, the main phytoalexin of peas (Yamada et al., 1989). Some suppressors isolated from germination fluid or culture fluid of phytopathogenic fungi have been reviewed by Shiraishi et al. (1994). To control certain diseases, it is very important to understand the infection mechanisms of the pathogen.

Pepper anthracnose is one of the major limiting factors in red pepper production. The yield loss is serious because pepper fruits exhibiting anthracnose have little market value. In Korea, the annual damage resulting from this di- 
sease has been estimated at more than US \$100 million, corresponding to approximately $10 \%$ of the total annual pepper production. Anthracnose creates lesions mainly on immature green and mature red fruits and leaves. Park and Kim (1992) reported that several Colletotrichum species, such as $C$. gloeosporioides, C. dematium, C. coccodes, and C. acutatum, and Glomerella cingulata were involved in the anthracnose symptoms, with $C$. gloeosporioides being the dominant pathogen. Recently, the major pathogen has been changed from C. gloeosporioides to C. acutatum, even though it has not been illustrated the changing reasons (Kim et al., 2008). However, it is unclear how C. acutatum is able to infect pepper fruits, and whether infection by $C$. acutatum is related to plant hormones. In the case of postbloom fruit drop caused by C. acutatum in citrus plants, an imbalance of some plant hormones in citrus flowers infected by $C$. acutatum may be involved in symptom development and young fruit drop (Lahey et al., 2004). Here we examined whether plant hormones are related to symptom development of pepper anthracnose caused by C. acutatum.

\section{Materials and Methods}

Collection and maintenance of isolates. Four isolates of Colletotrichum acutatum obtained from several plants were used. Isolates JC24, BAC02063, PECH10, and TCBNU3 were prepared by single-spore isolation from diseased red pepper, Chinese matrimony vine, peach, and tea trees, respectively. Each piece of diseased fruit or leaf was surface-sterilized with $2 \%$ sodium hypochlorite for $1 \mathrm{~min}$ and washed twice with sterile distilled water (SDW), and then placed on potato dextrose agar (PDA; Difco Laboratories, Detroit, MI) amended with $300 \mu \mathrm{g} / \mathrm{ml}$ of streptomycin sulfate. All plates were incubated at $25^{\circ} \mathrm{C}$ for 5 days to induce fungal growth. Following incubation, spores were harvested with sterile distilled water and washed twice with SDW. The spore concentration was adjusted to $1 \times 10^{6}$ conidia/ml, and $50 \mu \mathrm{l}$ of the spore suspension were spread on PDA amended with $300 \mu \mathrm{g} / \mathrm{ml}$ of streptomycin. Three days after incubation at $25^{\circ} \mathrm{C}$, mycelial discs at the margin of a small colony were cut and sub-cultured. All isolates were grown on PDA at $25^{\circ} \mathrm{C}$ and maintained on PDA slants at $4^{\circ} \mathrm{C}$ until used.

Preparation of inoculum of $C$. acutatum for the pathogenicity test. To prepare inoculum suspension, each isolate of C. acutatum was cultured on PDA for 7 days in the dark at $25^{\circ} \mathrm{C}$. Ten $\mathrm{ml}$ of SDW was poured into the culture, and filtered through four layers of cheesecloth to remove mycelial debris. The conidial suspension was washed twice in SDW by decanting the supernatant after centrifugation of the conidial suspension at $3,000 \times g$ for 15 min, then adjusted to $1 \times 10^{6}$ conidia/ml with a hemocytometer.

Effect of plant hormones on the pathogenicity of $\boldsymbol{C}$. acutatum. Effect of plant hormones ABA, gibberellic acid (GA), indole butyric acid (IBA), 6-benzylaminopurine (BA), and ethephon were evaluated on their pathogenicity toward several isolates of C. acutatum. In the laboratory, detached fruits harvested from pepper plants (cv. Nokkwang) grown in greenhouses were used for determining the pathogenicity of each isolate. Plant hormones were tank-mixed with a spore suspension of $C$. acutatum $\mathrm{JC} 24$, and the concentration was adjusted to 10,1 , and $0.1 \mathrm{mM}$ prior to application. Fruits were wounded by a pin used for measuring blood sugar (Accu-Chek ${ }^{\circledR}$ Softclix $^{\circledR}$ ) and inoculated by applying $5 \mu \mathrm{l}$ of a conidial suspension drop-wise to the wound with each concentration of plant hormone. Two wound sites were created per fruit. Inoculated fruits were placed in plastic containers $(30 \times 20 \times 10 \mathrm{~cm}, \mathrm{~W} \times \mathrm{L} \times \mathrm{H})$ containing three sheets of paper towel soaked in $100 \mathrm{ml}$ of distilled water to maintain high humidity $(>95 \% \mathrm{RH})$. Control fruits were inoculated with SDW with and without wounding. All treatments were performed in triplicate with ten pepper fruits. Lesion lengths were measured 1 and 2 weeks after inoculation to assess disease development. For investigating the effect of $\mathrm{ABA}$ on the development of anthracnose, a concentration of ABA adjusted to 0.0032, $0.016,0.08,0.4,2.0$, and $10.0 \mathrm{mM}$ in a conidial suspension of $C$. acutatum JC24 was applied to two sites on each fruit ( $5 \mu \mathrm{l}$ per site) with and without wounding. Storage of fruits in plastic containers maintained high humidity. The lesion lengths at the inoculation sites were measured every day beginning 3 days after inoculation.

Effect of ABA on the resistance of Capsicum species against pepper anthracnose. Detached fruits harvested from the pepper plants grown in greenhouses were used in the laboratory test. Capsicum annuum $\mathrm{cv}$. Jejujaerae and $C$. baccatum cvs. PBC 80 and $\mathrm{PBC} 81$ were used as susceptible and resistant plants to the anthracnose pathogen, respectively. For the inoculation of $C$. acutatum JC24, $2 \mathrm{mM}$ of ABA tank-mixed with conidial suspension adjusted to $1 \times$ $10^{6}$ conidia/ml was applied by dropping $5 \mu 1$ onto the wound sites. To assess disease development, disease incidence was investigated at each replicate 2 weeks after inoculation. Disease incidence was calculated as the percentage of inoculated sites per replicate that were diseased in relation to the total number of wound sites examined.

Effect of ABA on the pathogenicity of several isolates of C. acutatum against pepper fruit. The C. acutatum isolates JC24, BAC02063, PECH10, and TCBNU3 were 
obtained from diseased red pepper plants, Chinese matrimony vines, peach, and tea trees, respectively. Conidial suspensions were adjusted to $1 \times 10^{6}$ conidia/ml to which $2 \mathrm{mM}$ of ABA was added, and $5 \mu \mathrm{l}$ of the mixture were applied dropwise onto the wound sites of the fruit. Following 2 weeks of storage in a plastic humidity container, disease incidence was investigated for each replicate by calculating the percentage of the number of inoculated sites per replicate that were diseased in relation to the total number examined.

\section{Results}

Effect of plant hormones on the development of pepper anthracnose. To investigate the effect of five plant hormones on pepper anthracnose, lesion lengths of anthracnose were measured twice, 1 and 2 weeks after inoculation. ABA and GA showed a tendency of increased lesion length at the wound sites inoculated with $C$. acutatum JC24, whereas BA appeared to decrease the lesion length (Fig. 1). IBA and ethephon did not affect the symptom development of pepper anthracnose. Two weeks following inoculation, the largest lesion $(53.7 \mathrm{~mm})$ was observed after treatment with $1.0 \mathrm{mM}$ of ABA. Figure 2 shows the development of pepper anthracnose after treatment with ABA. However, there was no effect of ABA at sites inoculated without wounds. Lesion length rapidly increased 4 days after a 10 $\mathrm{mM}$ treatment of ABA to a fruit wound, reaching $38.7 \mathrm{~mm}$ in length after 10 days.

Effect of ABA on the resistance of Capsicum species to C. acutatum. Using detached fruits of Capsicum annum cv. Jejujaerae and C. baccatum cvs. PBC 80 and PBC81 as

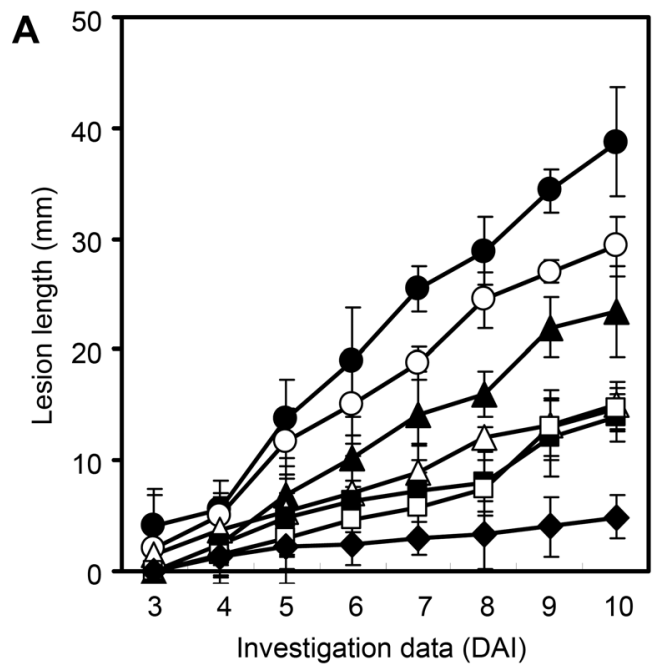

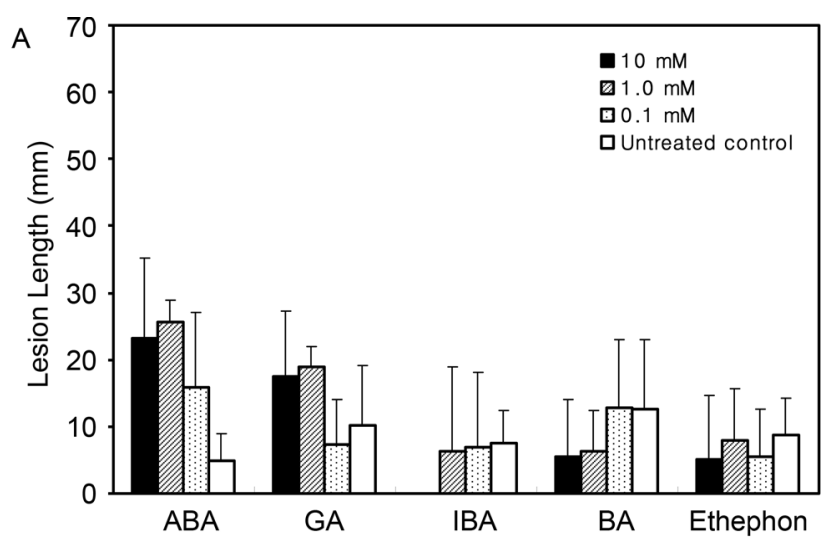

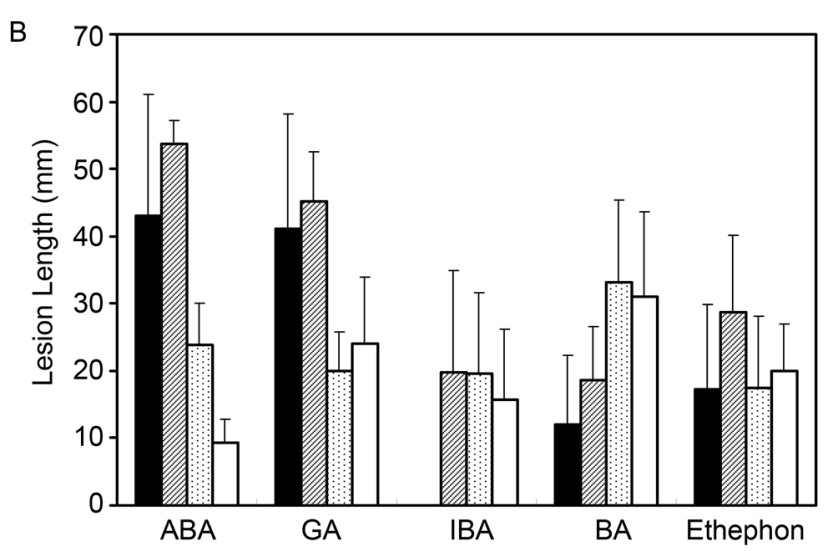

Fig. 1. Effect of plant hormones on the development of pepper anthracnose caused by Colletotrichum acutatum JC24. Plant hormones were applied simultaneously with a conidial suspension of $C$. acutatum JC24 adjusted to $1 \times 10^{6}$ conidia/ml by dropping onto wounded pepper fruits. The lesion lengths caused by pepper anthracnose were measured 1 (A) and 2 (B) weeks following inoculation.

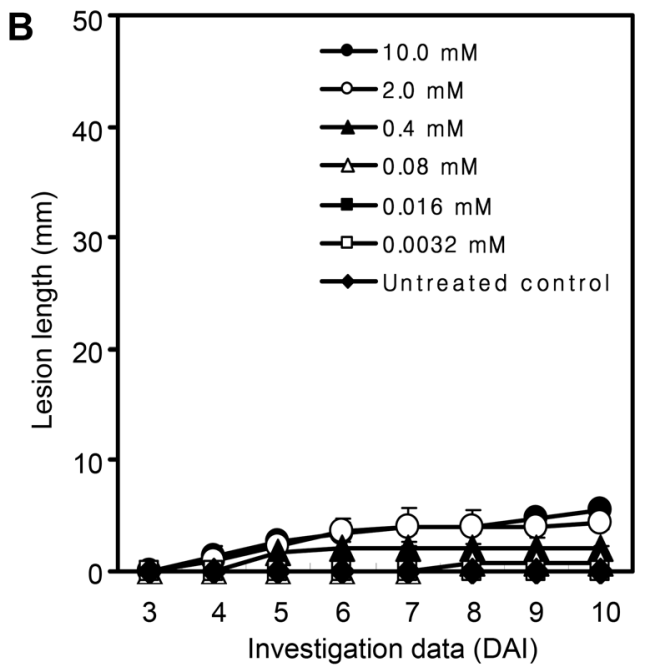

Fig. 2. Effect of abscisic acid (ABA) on the development of pepper anthracnose caused by Colletotrichum acutatum JC24. ABA was applied simultaneously with a conidial suspension of $C$. acutatum JC24 adjusted to $1 \times 10^{6}$ conidia/ml by dropping onto wounded (A) and intact pepper fruits (B). The lengths of the lesions caused by pepper anthracnose were continuously measured beginning 4 days after inoculation (DAI). 


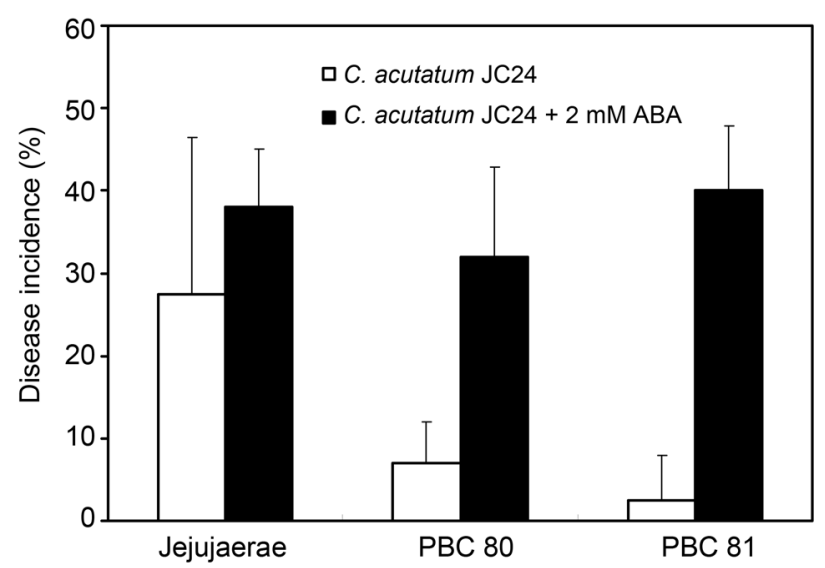

Fig. 3. Effect of abscisic acid (ABA) on the development of pepper anthracnose in fruits of the susceptible Capsicum annuum cultivar Jejujaerae, as well as the resistant Capsicum baccatum cultivars PBC 80 and PBC81. To prepare the inoculum, a conidial suspension of $C$. acutatum JC24 harvested from a 10-day culture was adjusted to $1 \times 10^{6}$ conidia $/ \mathrm{ml}$. Conidial suspensions with and without $2 \mathrm{mM}$ of ABA were dropped onto wounds of pepper fruits of susceptible and resistant cultivars. The disease incidence of pepper anthracnose was investigated 2 weeks after inoculation.

susceptible and resistant plants to the anthracnose pathogen, respectively, we investigated the effect of $\mathrm{ABA}$ on the resistance of Capsicum species to $C$. acutatum. When $C$. acutatum JC24 was inoculated onto a pepper wound site by dropping a conidial suspension adjusted to $1 \times 10^{6}$ conidia/ $\mathrm{ml}$, C. baccatum cvs. PBC 80 and PBC 81 showed resistant responses with disease incidences of 7.0 and $2.5 \%$, respectively. Jejujaerae was susceptible to $C$. acutatum JC24, showing a $27.5 \%$ disease incidence. However, when detached fruits were inoculated with a conidial suspension of $C$. acutatum JC24 including $2 \mathrm{mM}$ of ABA, the disease incidence of all cultivars of $C$. annuum and $C$. baccatum was increased by more than $30 \%$. Figure 4 shows the results of dropping $2 \mathrm{mM}$ of $\mathrm{ABA}$ alone onto wound sites, which did not induce any symptoms; even Jejujaerae, a cultivar susceptible to anthracnose, as well as the resistant cultivars PBC80 and PBC 81, were symptomless.

Effects of ABA on the pathogenicity of several isolates of C. acutatum toward pepper fruit. We examined the pathogenicity of four isolates of C. acutatum obtained from red pepper plants, peach trees, Chinese matrimony vine, and tea trees on $C$. annuum cv. Nokkwang. When each of the four isolates was administered without ABA, JC24 and

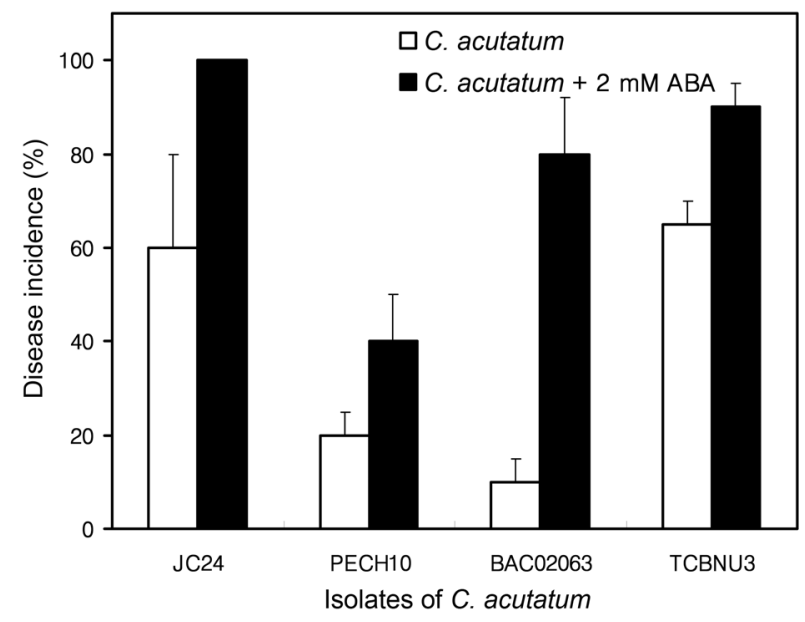

Fig. 5. Effect of abscisic acid (ABA) on the pathogenicity of Colletotrichum acutatum cultivar Nokkwang in pepper fruits. The four isolates of $C$. acutatum used in this study were obtained from pepper plants, Chinese matrimony vine, peach, and tea trees. To prepare the inoculum, conidial suspensions of each isolate of $C$. acutatum harvested from a 10-day culture were adjusted to $1 \times 10^{6}$ conidia/ml. Conidial suspensions with and without $2 \mathrm{mM}$ of ABA were dropped onto the wounds of pepper fruits. The disease incidence caused by pepper anthracnose was investigated 2 weeks after inoculation.
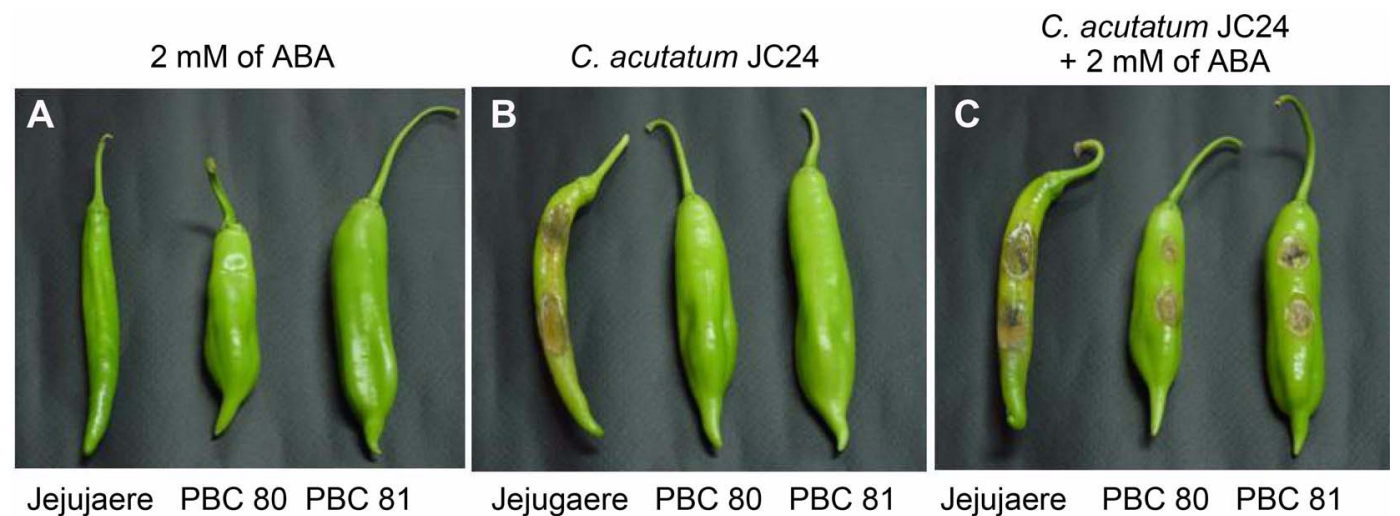

Fig. 4. Photographs of pepper anthracnose lesions on fruits of the susceptible Capsicum annuum cultivar Jejujaerae and the resistant Capsicum baccatum cultivars PBC 80 and PBC81. (A) A 2 mM dose of ABA was applied to each wound, (B) Each fruit was inoculated with Colletotrichum acutatum JC24 only, and (C) Each fruit was inoculated with a conidial suspension of C. acutatum JC24 with $2 \mathrm{mM}$ of ABA. 

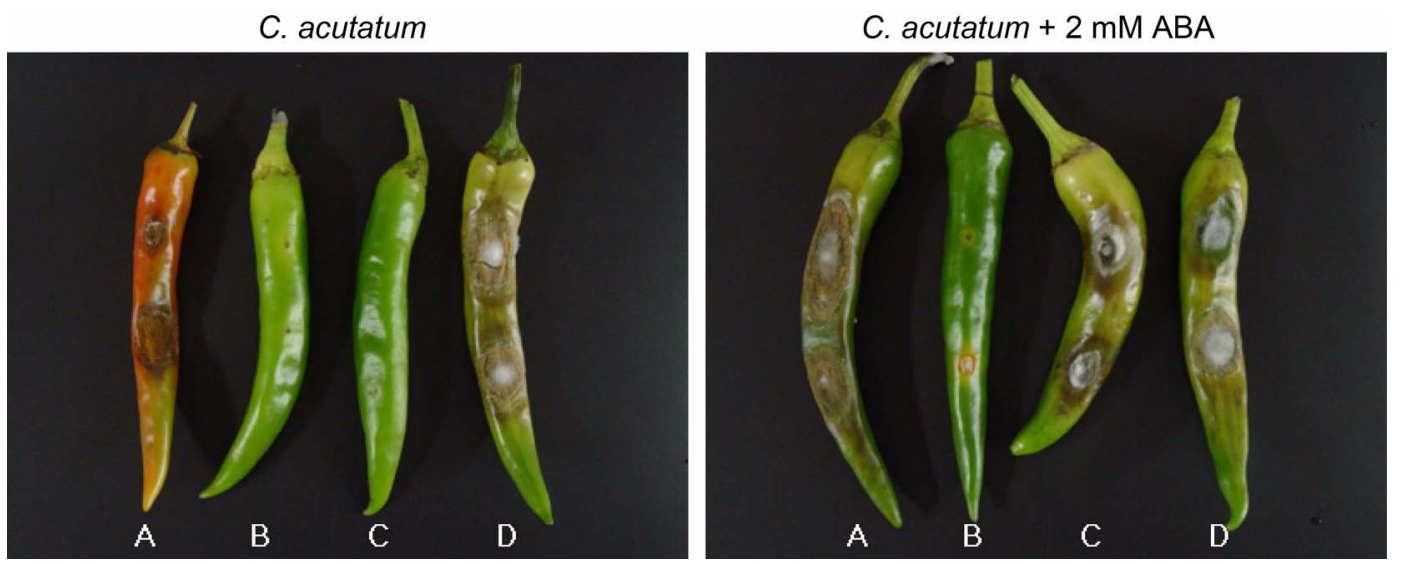

Fig. 6. Photographs of pepper anthracnose on pepper fruits of Colletotrichum acutatum cultivar Nokkwang. A: Colletotrichum acutatum JC24 isolated from pepper; B: C. acutatum PECH10 from peach; C: C. acutatum BAC02063 from the Chinese matrimony vine; D: $C$. acutatum TCBNU3 from tea plants.

TCBNU3 obtained from pepper and tea plants showed 60\% and $65 \%$ pathogenicity, respectively. Conversely, the disease incidence in PECH10 was $20 \%$, while that in BAC02063 was $10 \%$. However, the pathogenicity of all isolates of $C$. acutatum increased markedly when inoculated together with $2 \mathrm{mM}$ of ABA (Figs. 5 and 6). The disease incidence of C. acutatum BAC02063 isolated from the Chinese matrimony vine increased from $10 \%$ to $80 \%$ when inoculated with $2 \mathrm{mM}$ of ABA.

\section{Discussion}

We demonstrated that ABA had a significant effect on the pathogenicity of $C$. acutatum. Simultaneous inoculation of C. acutatum $\mathrm{JC} 24$ with $\mathrm{ABA}$ resulted in increased incidence of pepper anthracnose. Depending on the concentration of ABA administered, the anthracnose lesion length increased at the wound site of pepper fruits inoculated with a conidial suspension of $C$. acutatum JC24 with ABA. The simultaneous inoculation of $C$. acutatum JC24 with $2 \mathrm{mM}$ of ABA resulted in a change in the resistance response of Capsicum baccatum cv. PBC80 and PBC81, which were previously resistant to pepper anthracnose. With other isolates of $C$. acutatum obtained from Chinese matrimony vine, peach, and tea plants, ABA recovered the pathogenicity of $C$. acutatum that did not initially appear to infect pepper fruits.

ABA is one of the most important plant hormones known; thus, its action mechanisms have been studied extensively for decades. ABA appears to influence several physiological and developmental events (Busk, 1998; Leung and Giraudat, 1998). It plays important roles in the adaptation of vegetative tissues to abiotic environmental stresses such as drought and high salinity, as well as in seed maturation and dormancy (Jensen et al., 1996; McCarty, 1995). In addition, ABA suppressed the induction of systemic acquired resistance (SAR) in Arabidopsis by inhibiting the pathway both upstream and downstream of salicylic acid (SA), independently of the jasmonic acid/ethylene-mediated signaling pathway (Yasuda et al., 2008).

With Capsicum annuum cv. Jejujaerae susceptible to pepper anthracnose and C. baccatum cv. PBC80 and PBC81 resistant to it, Kim et al. (2004) reported that several mechanisms were involved in the defense of resistant pepper fruits against $C$. gloeosporioides infection, including programmed cell death (PCD) and cuticle thickening in epidermal cells related to resistant responses. Disease resistance in plants is often characterized by a rapid PCD. The local cell death confines the growth of the pathogen and is accompanied by a battery of defense responses that together result in the resistance of plants to pathogen attack. These induced defenses include the rapid production of reactive oxygen species (ROS) as well as nitric oxide, the local thickening of cell walls, and transcription-dependent mechanisms such as the production of hydrolytic enzymes and phytoalexins (Bowles, 1990; Brisson et al., 1994; Delledonne et al., 1998; Lamb and Dixon, 1997). Soybean cell cultures (cv. Williams 82) responded to Pseudomonas syringae expressing the avirulence gene AvrA with PCD of plant cells to pathogen attack. This PCD was reported to be under the control of SA via an unknown mechanism (Ludwing and Tenhaken, 2000). In the presence of low concentrations of SA, the cells showed very rapid cell death, which required only half of the time normally needed for the hypersensitive reaction (HR). Northern blot studies of defense-related genes showed that the expression of many of these genes was tightly linked to the status of the cell death program rather than to pathogen-derived elicitors. Thus, the expression was much faster in the SA-accelerated PCD than in the normal HR. In contrast, other pathogen-responsive genes were induced independently of the speed of PCD, 
indicating a divergent signaling mechanism. Recently, Yasuda et al. (2008) reported that treatment with ABA suppressed the induction of SAR in Arabidopsis using two types of SAR-inducing chemicals, 1,2-benzisothiazol-3(2H)one1,1-dioxide and benzo(1,2,3)thiadiazole-7-carbothioic acid S-methyl ester, which act upstream and downstream of SA, respectively, in the SAR signaling pathway. In an analysis using several Arabidopsis mutants in combination with these chemicals, treatment with ABA suppressed SAR induction by inhibiting the pathway both upstream and downstream of SA, independently of the jasmonic acid/ ethylene-mediated signaling pathway (Yasuda et al., 2008). $\mathrm{SAR}$ induction even by the $\mathrm{NaCl}$-activated environmental stress response proved to be dependently suppressed by ABA. In this study, the simultaneous application of ABA with the $C$. acutatum $\mathrm{JC} 24$ pathogen resulted in increased lesion length and disease incidence in pepper fruits and the development of typical anthracnose symptoms in fruits of C. baccatum cvs. $\mathrm{PBC} 80$ and $\mathrm{PBC} 81$ previously resistant to pepper anthracnose. These results indicate that $\mathrm{ABA}$ may suppresses defense mechanisms such as PCD and cuticle thickening at wound sites of fruits of $C$. baccatum cvs. PBC80 and PBC81 inoculated with C. acutatum JC24. Given that this defense-mechanism-suppressing effect of ABA also occurred in C. annuum cv. Nokkwang, which is normally susceptible to anthracnose, other isolates of $C$. acutatum showing a low pathogenicity to cv. Nokkwang were able to develop typical symptoms of anthracnose. We did not observe increased pathogenicity through inoculation of pepper fruits at non-wound sites. This may be because ABA was not absorbed into the epidermis of the pepper fruits. Future studies should concentrate on quantifying the changes in $\mathrm{ABA}$ at wound sites treated with the plant hormone, and should examine the role of $\mathrm{ABA}$ in the development of pepper anthracnose.

When the lethal yellowing of coconut palms associated with mycoplasma-like organisms was examined, diseased coconut palms showed symptoms such as premature nut fall and inflorescence necrosis followed by leaf yellowing and leaf necrosis, and died within 3-6 months (PlavsicBanjac et al., 1972). The concentration of ABA in the leaves and the capacity of leaf tissue to form ethylene increased in affected palms, suggesting that a hormone imbalance occurred in diseased palms (León et al., 1996). Furthermore, treatment of healthy palms with an ethylenereleasing agent (ethephon) resulted in symptoms that mimicked some of the leaf yellowing of LY symptoms. However, our results indicated that healthy fruits of $C$. annuum cv. Jejujaerae and C. baccatum cvs. PBC 80 and PBC81 inoculated with $2 \mathrm{mM}$ of ABA did not exhibit typical symptoms of pepper anthracnose (Fig. 4). Also, ABA did not have an effect on increasing the mycelial growth of $C$. acutatum JC24 on PDA inoculated with $2 \mathrm{mM}$ of ABA (data not shown).

Several reports have shown that imbalances in plant hormones caused typical pathogen symptoms. For example, infection of citrus flower petals by $C$. acutatum triggered significant accumulation of IAA, ethylene, and jasmonic acid, supporting the role of these plant hormones in the development of young fruit drop and disease progression (Lahey et al., 2004). Inoculation of pepper fruits with $C$. acutatum $\mathrm{JC} 24$ might not trigger the accelerated accumulation of $\mathrm{ABA}$ at infection sites on fruits; however, exogenous $\mathrm{ABA}$ might result in suppressing the defense mechanisms of pepper fruits, followed by a change in the susceptibility of pepper fruits and the development of pepper anthracnose even on the fruits of resistant cultivars. Therefore, to understand the pathogenic mechanisms of $C$. acutatum leading to pepper anthracnose, the role of plant hormone imbalance at the infection sites and the suppression of plant defense-related gene expression by plant hormones should be examined.

\section{Acknowledgment}

This work was supported by a grant from BioGreen 21 Program, Rural Development Administration, Republic of Korea.

\section{References}

Bouarab, K., Melton, R., Peart, D., Baulcombe, D. and Osbourn, A. 2002. A saponin-detoxifying enzyme mediates suppression of plant defences. Nature 418:889-892.

Brisson, L. F., Tenhaken, R. and Lamb C. J. 1994. Function of oxidative cross-linking of cell wall structure proteins in plant disease resistance. Plant Cell 6:1703-1712.

Bowles, D. J. 1990. Defense-related proteins in higher plants. Annu. Rev. Biochem. 59:873-907.

Busk, P. K. and Pages, M. 1998. Regulation of abscisic acid induced transcription. Plant Mol. Biol. 37:425-435.

Delledonne, M., Xia, Y., Dixon, R. A. and Lamb, C. 1998. Nitric oxide functions as signal in plant disease resistance. Nature 394:585-588.

Elad, Y. 1988. Involvement of ethylene in the diseases caused by Botrytis cinerea in rose and carnation flowers and the possibility of control. Ann. Appl. Bot. 113:589-598.

Elad, Y. 1997. Responses of plants to infection by Botrytis cinerea and novel means involved in reducing their susceptibility to infection. Biol. Rev. 72:381-422.

Escobar, M. A., Civerolo, E. L., Summerfelt, K. R. and Dandekar, A. M. 2001. RNAi-mediated oncogene silencing confers resistance to crown gall tumorigenesis. Proc. Natl. Acad. Sci. USA 98:13437-13442.

Jensen, A. B., Busk, P. K., Figueras, M., Alba, M. M., Perachia, 
G., Messeguer, R., Goday, A. and Pages, M. 1996. Drought signal transduction in plants. Plant Growth Regul. 20:105-110.

Kim J., Park, S. Choi, W., Lee, Y. and Kim, H. T. 2008. Characterization of Colletotrichum isolates causing anthracnose or pepper in Korea. Plant Pathol. J. 24:17-23.

Kim, K.-H., Yoon, J.-B., Park, H.-G., Park, E. U. and Kim, Y. H. 2004. Structural modifications and programmed cell death of chili pepper fruit related to resistant responses to Colletotrichum gloeosporioides infection. Phytopathology 94:12951304.

Lahey, K. A., Yuan, R., Burns, J. K., Ueng, P. P., Timmer, L. W. and Kuang-Ren, C. 2004. Induction of phytohormones and differential gene expression in citrus flowers infected by the fungus Colletotrichum acutatum. Mol. Plant-Microbe Interact. 17:1394-1401.

Lamb, C. and Dixon, R. A. 1997. The oxidative burst in plant disease resitance. Annu. Rev. Plant Physiol. Plant Mol. 48:251275.

León, R., Santamaría, J. S., Alpizar, L., Escamilla, J. A. and Oropeza, C. 1996. Physiological and biochemical changes in shoot of coconut palms affected by lethal yellowing. New Phytol. 134:227-234.

Leung, J. and Giraudat, J. 1998. Ascisic acid signal transduction. Annu. Rev. Plant Physiol. Plant Mol. Biol. 49:199-222.

Ludwing, A. and Tenhaken, R. 2000. Defense gene expression in soybean is linked to the status of the cell death program. Plant Mol. Biol. 44:209-218.

Malonek, S., Bömke, C., Bornberg-Bauer, E., Rojas, M. C., Hedden, P., Hopkins, P. and Tudzynski, B. 2005. Distribution of gibberellin biosynthetic genes and gibberellin production in the Gibberella fujikuroi species complex. Phytochemistry 66:1296-1311.

Maor, R., Haskin, S., Levi-Kedmi, H. and Sharon, A. 2004. In planta production of indole-3-acetic acid by Colletotrichum gloeosporioides f. sp. aeschynomene. Appl. Environ. Microbiol. 70:1852-1854.
Martin-Hernandez, A. M., Dufresne, M., Hugouvieux, V., Melton, R. and Osbourn, A. 2000. Effects of targeted replacement of the tomatinase gene on the interaction of Septoria lycopersici with tomato plants. Mol. Plant-Microbe Interact. 13:13011311.

McCarty, D. R. 1995. Genetic control and integration of maturation and germination pathways in seed development. Аnпu. Rev. Plant Physiol. Plant Mol. Biol. 46:71-93.

Park, K. S. and Kim, C. H. 1992. Identification, distribution and etiological characteristics of anthracnose fungi of red pepper in Korea. Korean J. Plant Pathol. 8:61-69.

Plavsic-Banjac, B., Hunt, P. and Maramorosch, K. 1972. Mycoplasma-like bodies associated lethal yellowing disease of coconut palms. Phytopathology 62:298-299.

Robinson, M., Riov, J. and Sharon A. 1998. Indole-3-acetic acid biosynthesis in Colletotrichum gloeosporioides f. sp. aeschynomene. Appl. Environ. Microbiol. 64:5030-5032.

Shiraishi, T., Yamada, T., Saitoh, K., Kato, T., Toyoda, K., Yoshioka, H., Kim, H.-M., Ichinose, Y., Tahara, M. and Oku, H. 1994. Suppressors determinants of specificity produced by plant pathogens. Plant Cell Physiol. 35:1107-1119.

Tudzynski, B. 2005. Gibberellin biosynthesis in fungi: genes, enzymes, evolution, and impact on biotechnology. Appl. Microbiol. Biotechnol. 66:597-611.

Yamada, T., Hasnimoto, H., Shirashi, T. and Oku, H. 1989. Supression of pisatin, phenylalanine ammonia-lyase mRNA, and ahalcone synthase mRNA accumulation by a putative pathogenicity factor from the fungus Mycosphaerella pinodes. Mol. Plant Microbe Interact. 2:256-261.

Yasuda, M., Ishikawa, A., Jikumaru, Y., Seki, M., Umezawa, T., Asami, T., Maruyama-Nakashita, A., Kudo, T., Shinozaki, K., Yoshida, S. and Nakashita, H. 2008. Antagonistic interaction between systemic acquired resistance and the abscisic acidmediated abiotic stress response in Arabidopsis. Plant Cell 20:1678-1692. 